\title{
Three Case Reports of Donor Site Iliac Bone Fracture as a Complication of Anterior Cervical Fixation Surgery
}

\author{
Taigo Kawaoka, Junya Hanakita, Toshiyuki Takahashi, and Mizuki Watanabe
}

\begin{abstract}
Although intervertebral implants have been advocated for cervical fixation surgery, classic Cloward's method or Smith-Robinson's methods using autologous iliac bone has been performed in our clinic for about 30 years. In most cases, the postoperative clinical course is uneventful with satisfactory result. However, the authors experienced three cases of donor site iliac bone fracture in the patients who performed anterior cervical fixation surgery. All three patients were female, elderly, short, and lean with low bone mineral density. Iliac bone fractures manifested with sudden pain in the vicinity of donor site. For the iliac bone fracture, two patients received invasive plate fixation surgery and the other was managed conservatively. As to the mechanism of donor site iliac bone fracture, the share stress during bone donation is speculated to cause iliac crest fragile. After beginning of walking after surgery, tractive force of the muscles attaching to the iliac crest play an important role in promoting the fracture cleft. Several clinical factors were analyzed to investigate the cause and prevention of this troublesome complication of cervical anterior fixation surgery. Age, female gender, lean, and small structure were risk factors for such iliac donor site fracture.
\end{abstract}

Keywords: anterior cervical fusion, iliac fracture, autograft

\section{Case Reports}

For the anterior cervical fixation surgery, intervertebral implants have often been used. However, in our clinic the classic Cloward's method or Smith-Robinson's method using autologous iliac bone graft have been adopted for more than 30 years.

As to the complications of the donor site iliac bone, besides donor site pain, local hematoma formation, infection, lateral femoral cutaneous nerve injury, incisional ventral herniation, and iliac bone fracture have been reported. ${ }^{1-19)}$ In the present article, the authors described three cases of iliac bone fracture in detail and discussed the mechanism and prevention of this rare complication.

\section{Case Summary}

\section{Case 1}

The patient was a 70-year-old woman [height, $150 \mathrm{~cm}$; body weight, $42 \mathrm{~kg}$; body mass index (BMI), $18.6 \mathrm{~kg} / \mathrm{m}^{2}$;

The Spinal Disorders Center, Fujieda Heisei Memorial Hospital, Fujieda, Shizuoka

Received: November 4, 2014; Accepted: February 25, 2015 young adult mean (YAM), 60\%; bone mineral density (BMD; $\left.\mathrm{L} 2-4)=0.543 \mathrm{~g} / \mathrm{cm}^{2}\right]$.

Chief complaints were numbness from both shoulders, extending down the arms, disturbance of skilled movements, and gait disorder.

These complaints appeared about 1 year prior to presentation at a local clinic. She began to receive symptomatic treatment at the local clinic. There was no improvement in her condition, and she was thus referred to our hospital for surgery.

Neurologically, muscle weakness below the right deltoid muscle and [manual muscle testing (MMT) 4/5] and that below the right iliopsoas muscle (MMT 4/5) were noted. In addition, increased deep tendon reflexes of the extremities were noted, but there was no evident sensory disorder.

Dynamic plain radiographs revealed $3 \mathrm{~mm}$ instability at the C3-4 levels. Magnetic resonance imaging (MRI) disclosed disc protrusion and changes in the intramedullary signals at the C3-4 levels.

Employing the Smith-Robinson procedure, we carried out anterior decompression, autologous iliac bone grafting, and anterior cervical fusion with a plate at the $\mathrm{C} 3-4$ levels.

Iliac bone was harvested with an osteotome.

Postoperative course was favorable; finger numbness was alleviated and walking improved over time. However, on Day 6 of surgery, when returning from the toilet to the ward at night, the patient experienced sudden pain on the lateral side of the right thigh, making it difficult to walk. Diagnostic imaging revealed a fracture of the superior anterior iliac spine, beginning at the donation site (Fig. 1A, B).

Because conservative treatment with rest was ineffective, pelvic reduction surgery was performed on Day 17 (Fig. 1C, D). Postoperatively, loading was avoided for about 1 month. She was discharged home on Day 76.

\section{Case 2}

The patient was a 77-year-old woman (height, $147 \mathrm{~cm}$; body weight, $43 \mathrm{~kg}$; BMI, 19.8\%, YAM, 61\%, BMD (L2-4) $=0.395 \mathrm{~g} / \mathrm{cm}^{2}$ ).

Chief complaints were weakness of the left hand and dizziness while walking.

The patient had been aware of these complaints for approximately 1 year prior to presentation. The symptoms had gradually worsened and she thus consulted our hospital.

Neurologically, muscle weakness was absent but she had increased deep tendon reflexes of the extremities, severe disturbance of skilled movements, and a spastic gait. 


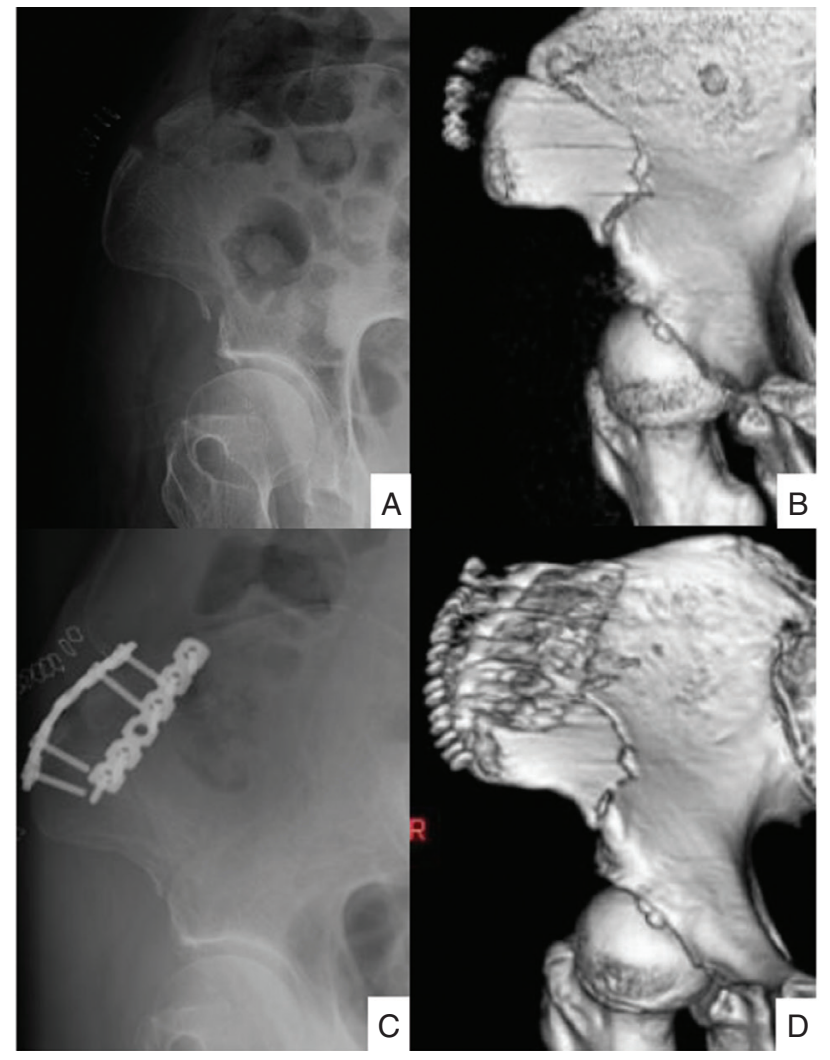

Fig. 1 C3-4 anterior fusion and plate fixation by Smith-Robinson's method. A, B: Fracture of the anterior superior iliac spine. C, D: Plate fixation of the anterior superior iliac spine.

Dynamic plain radiographs revealed instability at the narrowest site at the C3-4 levels. MRI disclosed ossification of the posterior longitudinal ligament at the $\mathrm{C} 4-5$ levels, compressive deformation of the spine at the $\mathrm{C} 3-5$ levels, and changes in intramedullary signals at the same levels.

Employing the Smith-Robinson procedure, we carried out anterior decompression, autologous iliac bone grafting, and anterior cervical fusion with a plate at the $\mathrm{C} 3-5$ levels.

Iliac bone was harvested with an osteotome.

The patient complained of pain at the donor site after surgery, but the pain was not severe enough to prevent walking. On Day 10, she was discharged home, walking without assistance. She resumed normal activities of daily living. On Day 14 , however, pain and hematoma suddenly appeared at the bone donor site, making it difficult to walk. She thus visited the outpatient critical care unit that night.

Diagnostic imaging revealed a superior anterior iliac spine fracture and an iliac crest fracture (Fig. 2A, B).

On Day 25, iliac bone reduction with fusion surgery was performed. On Day 85, she was discharged home, walking without assistance (Fig. 2C, D).

\section{Case 3}

The patient was an 80-year-old woman (height, $146 \mathrm{~cm}$; body weight, $39.5 \mathrm{~kg}$; BMI 18.5\%; YAM, 68\%, BMD (L2-4) $=0.609 \mathrm{~g} / \mathrm{cm}^{2}$ ).

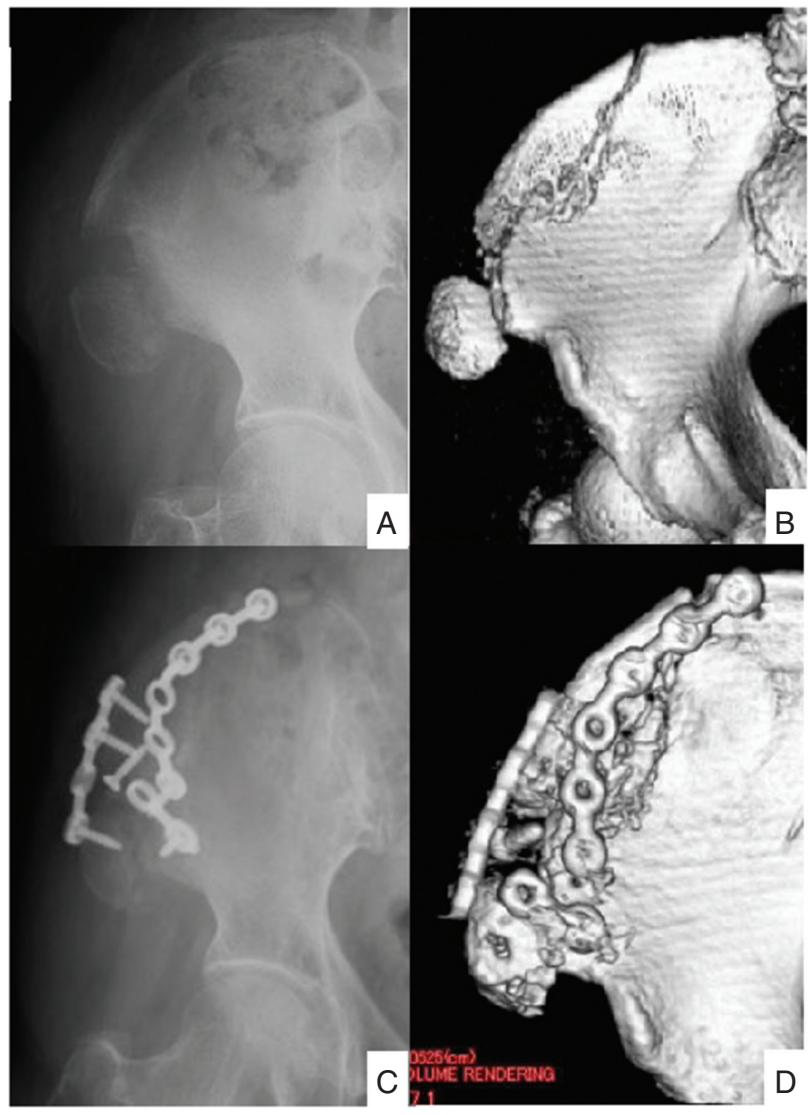

Fig. 2 C3-5 anterior fusion and plate fixation by Smith-Robinson's method. A, B: Fracture of the anterior superior iliac spine and the iliac crest. C, D: Plate fixation of the anterior superior iliac spine and the iliac crest.

The chief complaint was numbness of the distal extremities.

She had begun to be aware of distal extremity numbness about 1 year prior to presentation. Disturbance of skilled movements had gradually worsened, making it difficult to use chopsticks, accompanied by the appearance of a spastic gait. She was thus referred to our hospital.

Neurologically, increased deep tendon reflexes of the extremities, disturbance of skilled movements, and spastic gait were noted.

Dynamic plain radiographs revealed instability at the $\mathrm{C} 4-5$ levels. MRI disclosed local kyphosis at the same level, accompanied by spinal cord flattening and changes in intramedullary signals.

The patient underwent C3-6 laminectomy and C3-6 posterior fusion, and anterior decompression with autologous iliac bone grafting employing the Cloward procedure.

The donor iliac bone was harvested with a reamer designed specifically for the Cloward procedure.

On Day 1 of surgery, the patient began to complain of pain at the bone donor site during positional changes. On Day 5, when full-scale rehabilitation was started at the bed side, the patient complained of sudden severe pain at the bone donor site. 


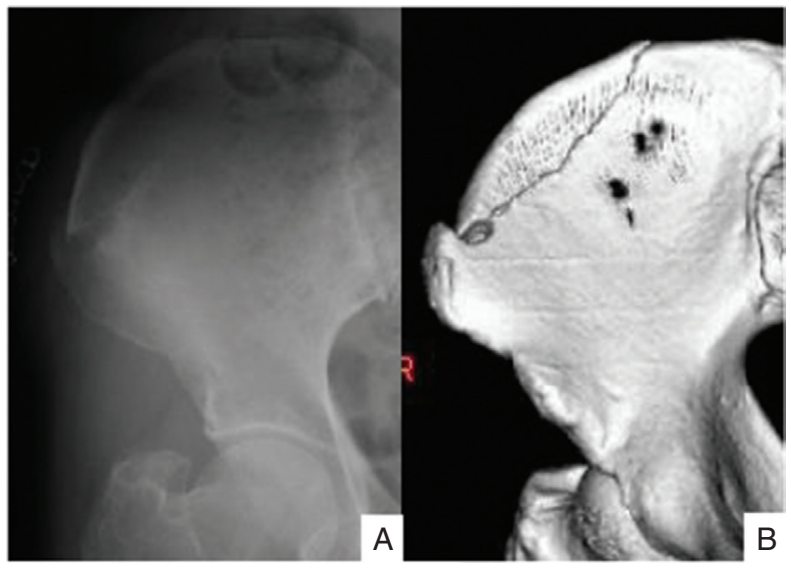

Fig. 3 C3-6 Laminectomy, posterior fixation, and C4-5 anterior fusion by Cloward's method. A, B: Fracture of the iliac crest.

Diagnostic imaging revealed an iliac crest fracture, extending posteriorly from the bone donor site (Fig. 3A, B).

After the onset of iliac crest fracture, the patient minimized movement. Bone fragment dislocation did not further advance and the pain gradually resolved. After activates of daily living had been restored to a satisfactory level, the patient was discharged home on Day 59, walking without assistance.

\section{Analysis of the Cases of the Cervical Anterior Fixation Surgery at Our Department}

In principal, anterior cervical surgery by Cloward's method is adopted in our department. When the patients show preoperative cervical instability, Smith-Robinson's method reinforced by cervical anterior plating or posterior fixation is performed. Between January 2005 and November 2012, 188 patients received anterior cervical fixation surgery in our department. The clinical data were analyzed in detail.

There were 135 men and 53 women. Mean age at surgery was 58.0 years for men and 57.6 years for women. Mean height was $168.4 \mathrm{~cm}$ for men and $154.4 \mathrm{~cm}$ for women. Mean body weight was $67.1 \mathrm{~kg}$ for men and $54.3 \mathrm{~kg}$ for women. Mean BMI was 23.6 for men and 22.8 for women. The mean BMD of the cases with fractures was $0.486 \mathrm{~g} / \mathrm{cm}^{2}$ (range: $0.395-0.658 \mathrm{~g} / \mathrm{cm}^{2}$ ). Mean YAM was $63 \%$ (range: $60-68 \%$; $70 \%$ or less is taken to indicate osteoporosis). BMD measurements had not been obtained for most of the patients free of iliac bone fractures.

Among the three patients with donor site iliac bone fractures, the bones were harvested with an osteotome in two cases of Smith-Robinson's method and with a reamer specially designed for another case of Cloward's procedure.

Pain at the bone donor site developed suddenly in all three cases, resulting in marked disturbance of walking. The mean duration from cervical fusion surgery until the onset of iliac bone fracture was 9.7 days (range: 5-17 days). Of the three cases with fractures, two underwent invasive plate fusion and one was managed conservatively. The mean period from cervical surgery until discharge from the hospital was 70.3 days (range: 59-85 days).

\section{Discussion}

Recently, several kinds of cervical implants such as titanium, ceramic carbon, or synthetic resin have often been used for cervical anterior fixation surgery. The reasons for using such implants seem to achieve rapid and firm fixation. Another possible reason for such implants is to avoid various problems in the site of autologous bone graft donation. Donor site pain is the most famous complication, the incidence of which ranges from $3.5 \%$ to $27 \%$ has been reported. However, Ikeda et al. ${ }^{9)}$ described that donor site pain is temporary and no cases last longer than 3 months. They advocated the superiority of autologous iliac bone graft because of good bone union and low cost compared with artificial materials.

In our study, there was no infection or hematoma. Although permanent donor site pain or outside femoral cutaneous neuralgia occurred in $3.2 \%$, almost all patients did not have a pain to interfere their daily life. In our department, iliac bone graft has been used for more than 30 years with satisfactory result. However, the authors experienced three cases of iliac bone fracture. We investigate the factors, mechanism, and protection of this iliac donor site fracture to perform cervical anterior fixation surgery of Cloward's and Smith-Robinson's method more safely and more satisfactorily.

\section{Frequency of donor site iliac bone fracture}

According to literature, five articles have been reported with the mean incidence being $1.7 \%$ (range: $0.4-3.5 \%$ ). ${ }^{2,7,17-19)}$ In our series, the incidence was $1.6 \%$ (3/188 patients).

\section{Form of iliac bone fracture}

The fracture could be divided into three types (Fig. 4):

Type A: Fracture line runs anteroinferiorly

Type B: Fracture line runs posteriorly, running along the iliac crest

Type C: Mixed fracture line of Type A and Type B

All the fractures described in the eight prior reports were of Type A., ${ }^{1,2,6,7,10,15,17-19)}$

3. Comparison of bone fracture cases with non-fracture cases

Among the 188 patients who received anterior fixation surgery in our department, there were 53 women free of iliac bone fractures. Various factors were compared between these 53 women and our 3 patients who had experienced factures.

In the 53 female patients, Cloward's method was adopted in 36 cases and Smith-Robinson's method in 17 cases. In the 36 patients treated by Cloward's method, mean age was 57.6 years, mean height was $154.4 \mathrm{~cm}$, mean body weight was $54.3 \mathrm{~kg}$, and mean BMI was $22.8 \mathrm{~kg} / \mathrm{m}^{2}$. In the 17 patients treated by Smith-Robinson's method, mean age was 56.1 years, mean height was $154.6 \mathrm{~cm}$, mean body weight was $54.2 \mathrm{~kg}$, and mean BMI was $22.6 \mathrm{~kg} / \mathrm{m}^{2}$.

On the other hand, among the three cases of complicated fracture, Cloward's method is one case, Smith-Robinson's method was two cases. The case of Cloward's method; age 


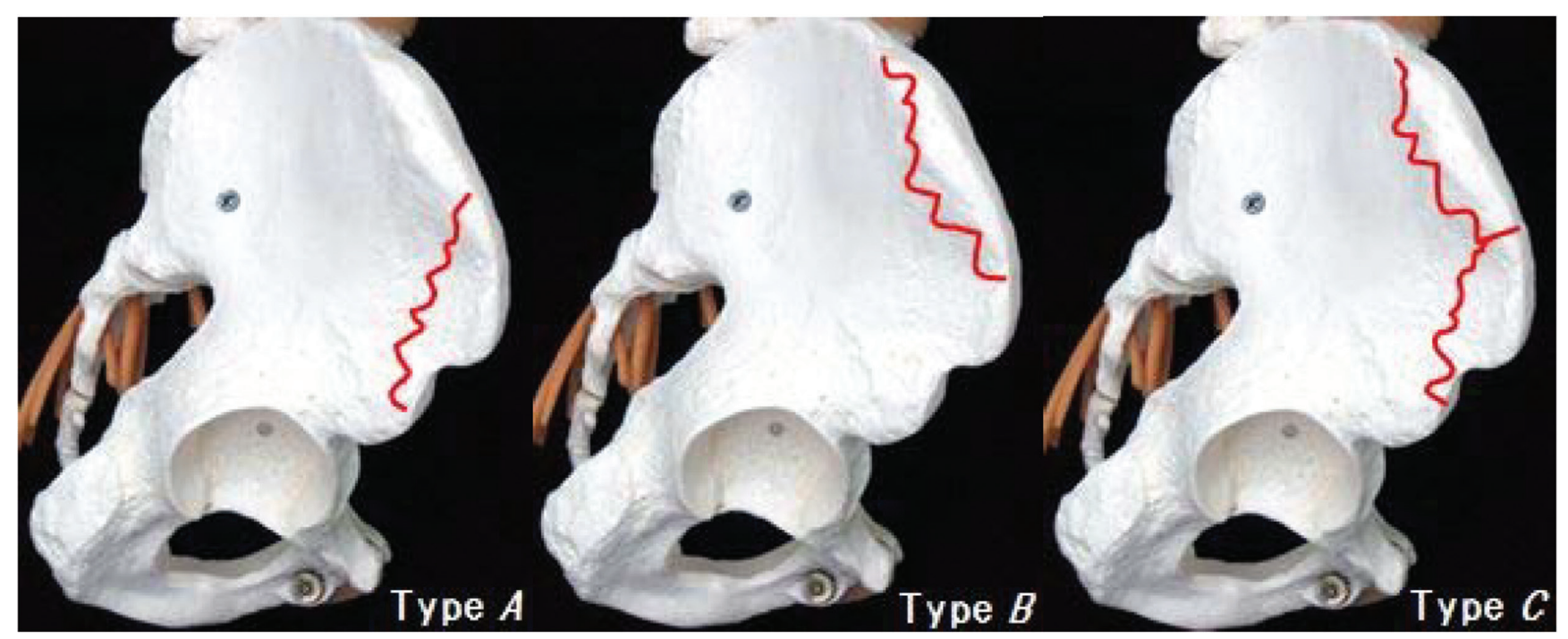

Fig. 4 Classification of the iliac fracture. Type A: Fracture of the front part from the anterior superior iliac spine. Type B: The fracture line extends backward from anterior superior iliac spine, including the iliac crest. Type C: Type A plus B.
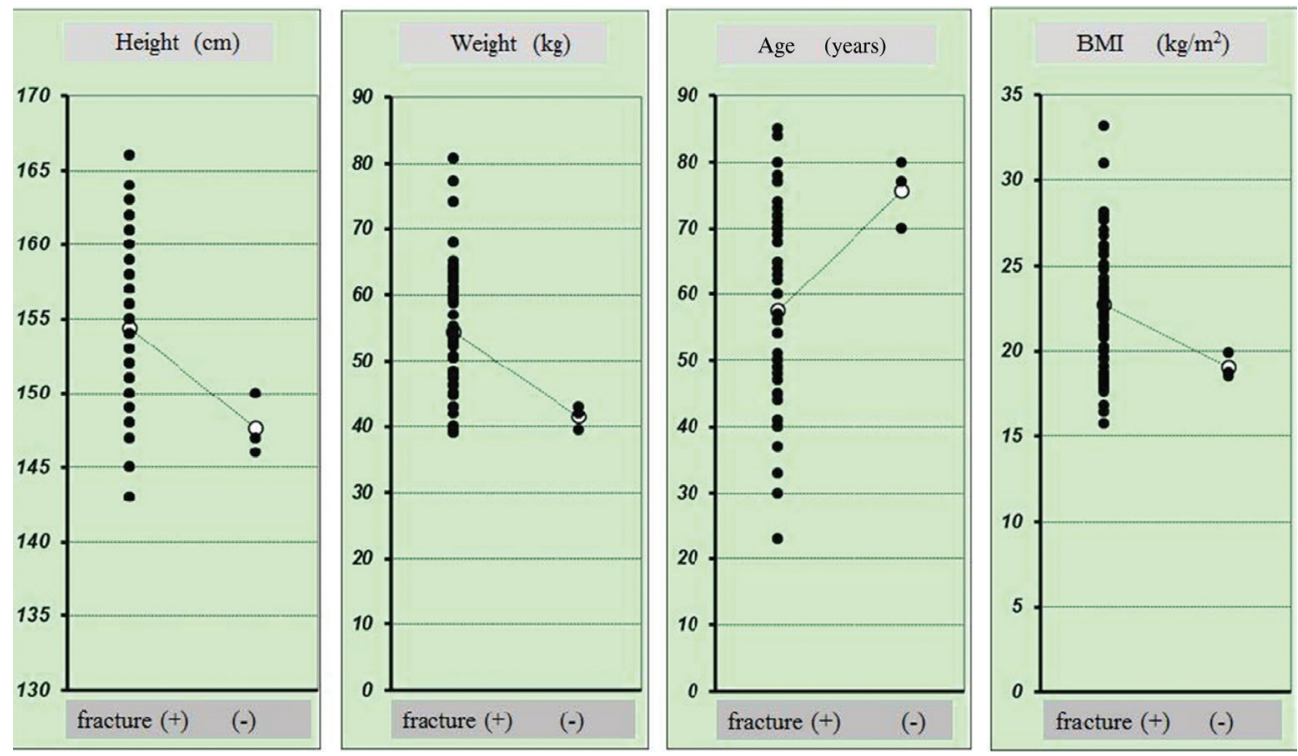

Fig. 5 Height, weight, age, and body mass index in the non-fracture group and fracture group.

Table 1 Parameters of the Cloward's method and Smith-Robinson's method, in the non-fracture group and fracture group

\begin{tabular}{lccc}
\hline Methods & & Non-fracture & Fracture \\
\hline Cloward & age (years) & 57.6 & 80 \\
& height $(\mathrm{cm})$ & 154.4 & 146 \\
& body weight $(\mathrm{kg})$ & 54.3 & 39.5 \\
& BMI $\left(\mathrm{kg} / \mathrm{m}^{2}\right)$ & 22.8 & 18.5 \\
Smith-Robinson & age (years) & 56.1 & 73.5 \\
& height $(\mathrm{cm})$ & 154.6 & 148.5 \\
& body weight $(\mathrm{kg})$ & 54.2 & 42.5 \\
& BMI $\left(\mathrm{kg} / \mathrm{m}^{2}\right)$ & 22.6 & 19.2 \\
\hline
\end{tabular}

BMI: body mass index. was 80 years, height was $146 \mathrm{~cm}$, body weight was $39.5 \mathrm{~kg}$, and BMI was $18.5 \mathrm{~kg} / \mathrm{m}^{2}$. Two cases of Smith-Robinson's method; mean age was 73.5 years, mean height was 148.5 $\mathrm{cm}$, mean body weight was $42.5 \mathrm{~kg}$, and mean BMI was $19.2 \mathrm{~kg} / \mathrm{m}^{2}$ (Table 1).

In the three patients with fractures, mean age was 75.7 years, mean height was $147.7 \mathrm{~cm}$, mean body weight was 41.5 $\mathrm{kg}$, and mean BMI was $19.0 \mathrm{~kg} / \mathrm{m}^{2}$. Thus, female gender and small frame were identified as significant characteristics of patients sustaining $(\mathrm{P}<0.05)$ fractures postoperatively (Fig. 5).

4. Mechanism of iliac bone fracture

Regarding the factors responsible for the post-donation iliac bone fracture, the damage to the iliac bone by the osteotome seems to play an important role. When the 
osteotome is applied, various types of cracks can occur in the iliac bone. ${ }^{7)}$ In case of minute cracks in the iliac bone, contraction force of the various muscles attached to the iliac bone could lead and accelerate the fractures of the damaged iliac bone. $\mathrm{Hu}$ et al. ${ }^{8)}$ measured the strength of the tension due to tailor muscle contraction in cadavers. Fractures primarily attributed to tailor muscle tension results in Type A fracture shown in Fig. 4, and Types B and C fractures could be brought by the contraction of the middle gluteal muscle.

According to the literature, all the reported cases were the iliac bone fracture. To the contrary, one of the present cases was noticed in the Cloward's method.

Although the true mechanism of such iliac bone fracture by the Cloward's reamer is not clear, the fact that such iliac bone fracture can happen should be taken into consideration even when the Cloward's method is performed.

5. Countermeasures for prevention of iliac bone fracture

When bone harvesting is performed in lean, elderly women, particular care and countermeasures should be paid. Regarding the use of an osteotome, it should be applied at right angles to the iliac bone cortex and the blade should be hit gently. As an alternative, tool bone saw could be applied for donor site graft formation.

\section{Conclusion}

Three cases of post-donation iliac bone fractures were described in detail. Fractures were classified into three types, the mechanism of which was discussed.

\section{Conflicts of Interest Disclosure}

No grants, technical support, or corporate support were received.

\section{References}

1) Al-Sayyad MJ, Abdulmajeed TM: Fracture of the anterior iliac crest following autogenous bone grafting. Saudi Med J 27: 254-258, 2006
2) Asano M, Fuchiya T, Nakahata K, Kizawa T, Fujiwara K: Perioperative complications of cervical spinal surgery. Cent Jpn J Orthop Traumat 48: 469-470, 2005

3) Banwart JC, Asher MA, Hassanein RS: Iliac crest bone graft harvest donor site morbidity. A statistical evaluation. Spine 20: 1055-1060, 1995

4) Blakemore ME: Fractures at cancellous bone graft donor sites. Injury 14: 519-522, 1983

5) Fernando TL, Kim SS, Mohler DG: Complete pelvic ring failure after posterior iliac bone graft harvesting. Spine 24: 2101-2104, 1999

6) Guha SC, Poole MD: Stress fracture of the iliac bone with subfascial femoral neuropathy: unusual complications at a bone graft donor site: case report. Br J Plast Surg 36: 305-306, 1983

7) Hu RW, Bohlman HH: Fracture at the iliac bone graft harvest site after fusion of the spine. Clin Orthop Related Res 309: 208-213, 1994

8) Hu RW, Hearn T, Yang J: Bone graft harvest site as a determinant of iliac crest strength. Clin Orthop Related Res 310: 252-256, 1995

9) Ikeda T, Nakamura T, Fujimoto T, Kikuchi T, Takagi K: Morbidity at iliac bone graft donor sites. Orthop Surg Traumatol 49: 666-668, 2000

10) Jones AA, Dougherty PJ, Sharkey NA, Benson DR: Iliac crest bone graft. Osteotome versus saw. Spine 18: 2048-2052, 1993

11) Kuhn DA, Moreland MS: Complications following iliac crest bone grafting. Clin Orthop Related Res 209: 224-226, 1986

12) Mine N, Taniguchi Y, Kitano T, Mine M, Yoshida M: A case of conservative treatment for fracture at the iliac bone graft harvest site. Cent Jpn J Orthop Traumat 51: 257-258, 2008

13) Ovalioglu AO, Kilincer C, Ovalioglu TC, Simsek O: Avulsion fracture of the anterior iliac crest after bone graft harvest: case report and review of techniques, risk factors and treatment. Turk Neurosurg 21: 423-426, 2011

14) Porchet F, Jaques B: Unusual complications at iliac crest bone graft donor site: experience with two cases. Neurosurgery 39: 856-859, 1996

15) Reale F, Gambacorta D, Mencattini G: Iliac crest fracture after removal of two bone plugs for anterior cervical fusion. Case report. J Neurosurg 51: 560-561, 1979

16) Reynolds AF, Turner PT, Loeser JD: Fracture of the anterior superior iliac spine following anterior cervical fusion using iliac crest. Case report. J Neurosurg 48: 809-810, 1978

17) Satoh H, Tajima T, Yamakawa K, Sakamoto T, Sawaumi A, Oka T, Gotoh H: Fracture of the anterior superior iliac spine after anterior spinal fusion. Orthop Surg Traumatol 13: 1999-2001, 1982

18) Schnee CL, Freese A, Weil RJ, Marcotte PJ: Analysis of harvest morbidity and radiographic outcome using autograft for anterior cervical fusion. Spine (Phila Pa 1976) 22: 2222-2227, 1997

19) Tsubata O, Nishijima Y: Spinal fusion without bone grafting. Complications related to bone grafting and the countermeasures. J MIOS 6: 3-8, 1998

Corresponding author:

Taigo Kawaoka, MD, The Spinal Disorders Center, Fujieda Heisei Memorial Hospital, 123-1 Mizukami, Fujieda, Shizuoka 426-8662, Japan.

$\triangle$ taigo_maradona@yahoo.co.jp 